\title{
Mobile Internet telephony: mobile extensions to H.323
}

\author{
Wanjiun Liao \\ Department of Electrical Engineering \\ National Taiwan University \\ Taipei, Taiwan \\ Email:wjliao@cc.ee.ntu.edu.tw
}

\begin{abstract}
Internet telephony realizes the transmission of twoway and real-time traffic over IP-based networks. The dominant standard for Internet telephony is ITU-T Rec. H.323. With the current version of H.323, Internet telephony allows interoperability with circuit-switched telephone, but IP host mobility is not supported. In this paper, mobile extensions to H.323 that enable mobile Internet telephony service are proposed. The proposed approach combines the characteristics of both cellular phone system and mobile IP mechanism with Internet telephony, and therefore realizes the transmission of real-time voice traffic for both stationary and mobile hosts over IP-based networks. The most striking feature of the proposed approach is that the complicated mobility management functions are handled by the procedures for dynamically joining and departing from a conference, functions already defined in H.323. Therefore, our approach allows mobility support without the need for additional new entities, and with minimal modifications to existing H.323 standard. Such mobility extensions can serve as an add-on feature for the existing Internet telephony systems compliant to the H.323 standard.
\end{abstract}

Keywords: Internet telephony, H.323, Voice over IP, mobile Internet telephony, mobile extensions to H.323

\section{Introduction}

Internet telephony, also known as voice over IP or IP telephony, promises to transmit real time, two-way, synchronous voice traffic over the Internet or corporate Intranets. The dominant standard of Internet telephony is the International Telecommunication Union Telecommunication Standardization Sector (ITU-T) Recommendation H.323 [1, 2]. H.323 specifies technical requirements for multimedia communications, including the system components, control messages and functions for component communications, over packet switched networks. With H.323, data, voice, and video in an IP call are transmitted using the Real-time Transport Protocol [3].
Call setup and other call control signaling messages are carried out-of-band, sent through different paths other than those for the payload traffic.

In addition to IP telephony, another attractive phone service is toward mobility support, i.e., the ability to maintain communications while moving. This can be seen in the rapidly explosive usage of cellular phones. With the increasing popularity of PDA, lap-top computers, wireless LANs, and the desire for global connectivity, host mobility on the Internet has become an important issue. It may be desirable to integrate such mobility features to Internet telephony as well. For example, when a user originates a POTS phone call to an IP telephone on the Internet, the IP host may not be stationary or fixed. It is likely that the IP host is currently on the move from networks to networks, or the point of attachment of the IP host has changed. Like cellular phone services, mobile Internet telephony demands seamlessly roaming while conversation is in progress. Under the current version of H.323 service scope, however, host mobility is forbidden, resulting from the underlying IP mechanism that implicitly assumes that a host is fixed. Moreover, Voice over IP service is a real-time connection-oriented service over packet-switched IP-based networks. Neither the circuit-switched cellular phone approach nor the connectionless Mobile IP mechanism is capable of providing mobile Internet telephony service. New approaches are required for mobile IP telephony.

In this paper, an approach that enables mobile Internet telephony services from mobile extensions to H.323 is proposed. Through proper call setup signaling with the H.323 Gatekeeper, the address of the target endpoint (i.e., callee or called party) can be resolved before call establishment, enabling the service redirection to be completely handled in the application layer. Thus, it realizes mobile IP telephony services with IP. Our 
approach enables mobility support without the need for additional new entities and with minimal modifications to the H.323 standard, allowing such mobile IP telephony service to be a valued-added feature in the existing H.323-compliant Internet telephony systems.

The rest of the paper is organized as follows. The fundamentals and architecture of an mobile Internet telephony system extended from the $\mathrm{H} .323$ standard are described in Section 2. Call signaling procedures for mobility management are proposed in Section 3. Finally the concluding remarks are included in Section 4.

\section{Mobile Internet telephony}

In this section, mobile extensions to $\mathrm{H} .323$ for the provision of the mobile Internet telephony services are proposed. We will demonstrate how $\mathrm{H} .323$ provides an excellent environment for mobile extensions from Internet telephony. No extra system component is introduced. The definition of the H.323 terminal will be extended to refer to both stationary and mobile H.323 terminals, and the rest of the H.323 entities remain unchanged. Some terminology has been borrowed from Mobile IP for the system architecture. We try to reuse the existing protocols in IP networks and stick to those control messages and functions defined in Internet telephony mechanism as much as we can, and show how to seamlessly integrate mobility feature into H.323 compliant Internet telephony systems. We will first explain the concept of how to extend the mobility capability from the existing H.323 Internet telephony systems, and present system architecture and call signaling procedures for mobility management in the following sections.

\subsection{Internet telephony: from stationary to mobile terminals}

The Internet telephony was invented to enable realtime voice services for stationary IP hosts communicating with other stationary IP hosts on IP-based networks, or with circuit-switched telephones through an Internet Telephony Gateway (ITG). To provide host mobility in Internet telephony, roaming should be supported and handoff should be performed if necessary. A handoff allows a mobile terminal to be assigned to a new channel in the new location area. Upon crossing a region boundary, a handoff must be initiated; otherwise, the connection is broken and the ongoing conversation is interrupted. The question is what the granularity of the service area is and how handoff is handled.

In a cellular phone system, say GSM [4], a mobile phone requires a handoff upon crossing a cell boundary. The connection is handed off from the old cell to the new one. Once the mobile phone has been given a new channel in the new cell, the old channel in the old cell is released and made available to other user in that cell. Global roaming is realized through Home Location Register (HLR) and Visitor Location Register (VLR) that store the location information of mobile phones. HLR contains location information of users whose primary subscription area is in this service area, while VLR contains location information of users whose primary subscription is with another service area, but presently roaming in this area. When a location update is initiated for a visitor, the VLR sends the update to the HLR for further call processing. Note that location update in a mobility agent (HLR or VLR) is implicitly performed after a handoff for roaming across service area boundary in a connection-oriented cellular phone system.

In an IP telephony network with wired and wireless subnets, a mobile phone requires a handoff upon crossing a subnet boundary that causes the IP address of the mobile phone to change. Mobile IP [5] is currently the major standard for mobility management on the Internet. With the Mobile IP mechanism, roaming is performed through the cooperative support of home agent and foreign agent, similar to HLR and VLR in the GSM, respectively. When a location update is initiated, the foreign agent informs the home agent of the care-of address temporarily assigned to the visiting mobile host. This allows data destined to the mobile host in the foreign network to be routed correctly via IP tunneling. However; Mobile IP does not handle the handoff because it is based on a connectionless service model and lacks the concept of connection. IP telephony services, on the other hand, are of phone services and thus require connection-oriented service over packet-switched IP-based networks. Neither the circuit-switched cellular phone approach nor the connectionless Mobile IP mechanism enables mobile Internet phone service. New approaches are required for mobile IP phone services. 


\subsection{System architecture}

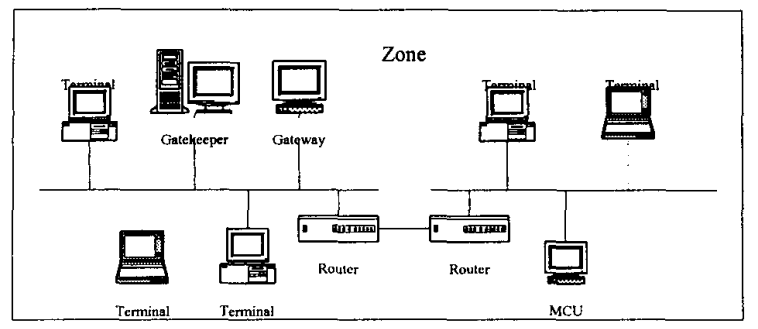

Fig. 1: A zone in the H.323

As defined in H.323, a zone is referred to as a collection of H.323 entities (terminal, ITG, Multipoint Control Unit) managed by a single Gatekeeper, as depicted in Fig. 1. Each zone contains exactly one Gatekeeper, but allows multiple subnets connected by routers. With the characteristics of only one Gatekeeper and multiple subnets, we observe that the zone plays a similar role to the service area of the cellular phone system, and the Gatekeeper, similar to the role of a mobility agent such as the HLR or VLR. The service area in a cellular phone system may consist of multiple cells and base stations under the management of a mobility agent. Similarly, a zone may consist of multiple subnets under the management of a Gatekeeper. When a mobile IP phone roams from subnet to subnet, the IP address of the IP phone is changed. The connection is broken and the ongoing conversation is interrupted unless a handoff is performed.

To allow roaming during a conversation, some terminology should be introduced. The home zone is defined as the zone that an H.323 terminal normally resides, and foreign zone, a zone that the H.323 terminal may visit. The corresponding Gatekeepers are called the home Gatekeeper and the foreign Gatekeeper, respectively. When a mobile host moves within a zone, it is referred to as intra-zone roaming. When it crosses the boundary to other zones, it is called inter-zone roaming. No location update in a Gatekeeper is required for intrazone roaming because a mobile terminal is still under the control of the same Gatekeeper. Location update is required only upon crossing a zone boundary. When roaming to a foreign zone, a visiting terminal notifies the visited foreign Gatekeeper of its existence with the transport address (a temporary IP address and a port number in a TCP/UDP environment) through registration.
A temporary IP address of the visitor may be acquired dynamically by such means as the Dynamic Host Configuration Protocol (DHCP) [6] in the respective subnet. The foreign Gatekeeper then informs the home Gatekeeper of the reachability information using the address of the affiliated foreign Gatekeeper as the care-of address of the visiting terminal, hiding the actual point of attachment of the visitor behind a coarser destination. The home Gatekeeper performs mobility binding which associates the home address of the moving terminal with the temporary care-of address. Such coarser mobility binding than Mobile IP reduces the number of location update messages when a terminal is roaming from subnets to subnets in a zone. Note that a Gatekeeper is responsible for address translation and call admission control services and may participate in call signaling, similar to a mobile switching center with the mobility agent in GSM. Recall that a mobile switching center is the heart of a GSM system that is responsible for connection establishment, management, and termination as well as call routing to proper cells. Therefore, global roaming can be realized by the cooperative support of home and foreign Gatekeepers.

\subsection{Mobility management}

Mobility management is the key to successfully enable mobile Internet telephony service over the connectionless IP networks. The core operations include registration, call establishment, roaming and handoff. In H.323/H.225.0 [7], an endpoint is registered to a Gatekeeper for admission to system access through an unreliable RAS channel. Call establishment and termination are performed before and after a call, respectively, through a reliable call signaling channel. However, as mentioned earlier, no mobility support is provided with the current version of H.323. Therefore, there is no mechanism for roaming and handoff handling, along with the registration and location update. We will demonstrate how to extend the capability of roaming and handoff from the existing H.323 standard.

When an H.323 terminal roams across different subnets once a connection has been established, thereby causing the IP address to change, the connection is broken unless it is handed off. Examine roaming scenarios shown in Fig. 2. TL1 denotes an H.323 terminal currently staying in subnet1, and TL2 denotes the same H.323 terminal roaming from subnet 1 to subnet 2 . The subnets are under 
the management of the same Gatekeeper in the case of intra-zone roaming as shown in Fig. 2 (a) and of different $\mathrm{G}$ itekeepers in inter-zone roaming as shown in Fig. 2 (b). In both cases, originally, there is only one connection cr :ated from the ITG to TL1. TL1 is communicating with th : ITG while roaming, and while the handoff is taking pl ice during the migration from the subnet1 to subnet2. $\mathrm{O}$ ice handoff is completed, the connection from the ITG to TL1 is terminated, and a new connection from the ITG to TL2 is established, leaving only one connection from th I ITG to TL2. The resources reserved and allocated for th 1 old connection in subnet 1 is released and made av ilable for reuse. Such a handoff simulates the handoff in the cellular phone system, that is, a new connection is fin it established and co-exists with the old connection, an 1 in turn the old connection is torn down.

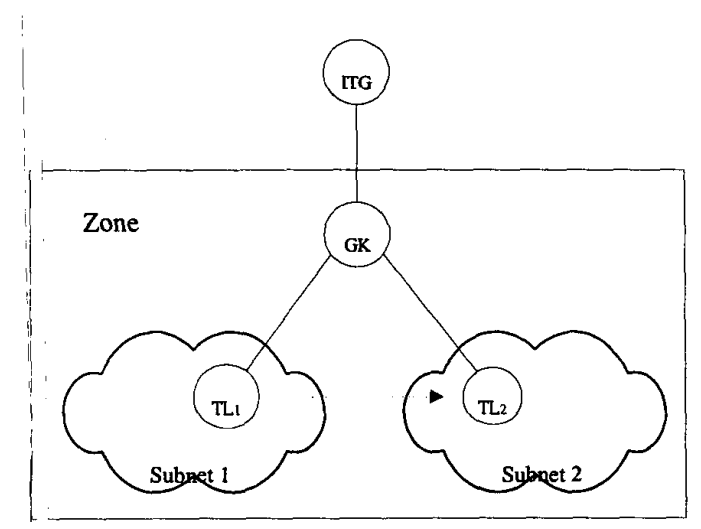

(a) Intra-zone roaming

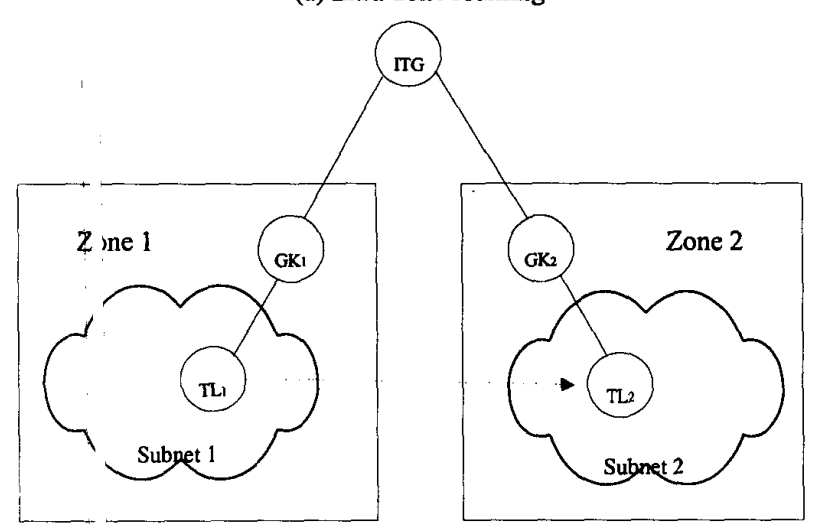

(b) Inter-zone roaming

Fig. 2: Roaming scenarios

Surprisingly, the procedure for handoff described abc re for mobile Internet telephony roaming may be real zed by the dynamic join and departure of an ad hoc multipoint conference in the H.323 compliant Internet telephony systems. An ad hoc multipoint conference is a point-to-point conference that can be expanded to multipoint conference at some time during the call [2]. The steps of dynamically joining and leaving an ad hoc conference are demonstrated in Fig. 3.

Originally, a point to point conference is first created between the ITG and TL1. TL2 then joins the conference either by being invited or by its own request through the MC in the ITG. A new connection from the ITG to TL2 is required to be setup when TL2 joins the conference, with the same media streams in the same conference. Finally, TL1 leaves the conference after TL2 has joined, resulting in the connection from the ITG to TL1 being torn down without affecting the media stream of the ITG to TL2 connection. This shows the effect of proposed handoff for the mobile Internet telephony equivalent to the dynamic join and departure of an ad hoc multipoint conference. Fig. 3 (a) depicts the ITG communicating with TL1 while roaming. Fig. 3 (b) depicts the handoff from the subnet 1 to subnet 2 while the conversation continues. Fig. 3 (c) shows that the handoff is nearly finished and the connection from the ITG to TL1 is broken. Finally, the handoff succeeds, and only the connection from the ITG to TL2 is left, as depicted in Fig. 3 (d).

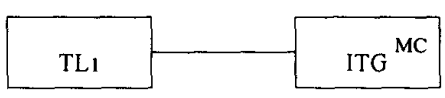

(a) Original conference

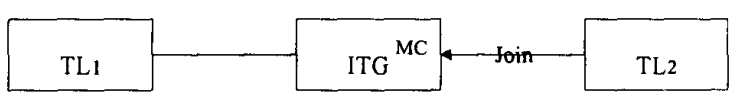

(b) TL2 joins the conference

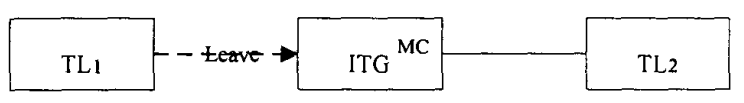

(c) TL1 leaves the conference

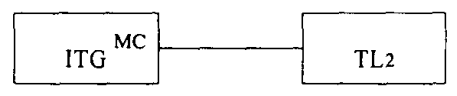

(d) Resultant conference

Fig. 3: Dynamic join and leave in a multipoint conference

The correspondence between mobility management and dynamic join and departure of an ad hoc conference 
strongly suggests the possibility of the support of mobile IP telephony service from H.323. Strikingly and interestingly, this approach extends stationary Internet telephony systems to seamlessly support mobile IP phone services, without any extra cost for such mobile extensions.

\section{Call signaling procedures for mobility management}

Because logical channel establishment for media transmission as defined in H.245 [8] remains unchanged after a call is established, the major focus of the following will be on the call signaling procedures for registration, connection establishment, and handoff for roaming. Due to the limited space, only a call made between a POTS phone and an H.323 terminal is demonstrated. The procedure is applicable for an $\mathrm{H} .323$ terminal to $\mathrm{H} .323$ terminal call as well.

Call handling can be divided into three parts: POTS phone to the ITG (on the PSTN), inside the ITG, and the ITG to H.323 terminal (on the Internet). Here the procedures for both POTS phone to the ITG and inside the ITG remain unchanged for the mobile extensions to Internet telephony services. Only the Internet part (i.e., between the ITG and H.323 terminal) is considered in this section. Again, due to the space limitation, only direct endpoint call signaling is demonstrated. The Gatekeeper routed call signaling can be derived in a similar manner.

\subsection{Registration}

An RAS (i.e., registration, admission, and status) channel defined in H.225.0 is used to convey messages for Gatekeeper discovery and endpoint registration. Gatekeeper discovery is a process used by an endpoint to ascertain which Gatekeeper to register with. In a mobile environment, a mobility agent may advertise its availability. Gatekeeper discovery will be extended and performed in two different ways: (1) a Gatekeeper may advertise its availability for mobility services, or (2) a mobile terminal may solicit the availability of a Gatekeeper. As defined in H.225.0, an endpoint may send a Gatekeeper Request (GRQ) message to the Gatekeeper well-known discovery multicast address to determine the
Gatekeeper, followed by the response from the Gatekeeper either with the Gatekeeper Confirmation (GCF) message to accept the registration, or with the Gatekeeper Reject (GRJ) for refusal. Therefore, in the first case, an extra message called Gatekeeper Advertisement (GAD) will be introduced. A Gatekeeper may advertise its availability with a GAD message through multicasting to the respective zone. In the second case, a mobile terminal may send a GRQ message to the Gatekeeper well known Discovery Multicast Address to ascertain the availability of a Gatekeeper, followed by the corresponding Gatekeeper's reply to the solicitation request with a GCF or GRJ.

As part of the configuration process, an endpoint will register with the Gatekeeper identified through the discovery process. Registration here refers as to the process by which an endpoint joins a Zone and informs the Gatekeeper of its existence with its address. This definition can be easily extended to the mobile environment in which registration is defined as a process whereby a mobile phone may exchange its current binding information with its home Gatekeeper. Registration should occur before any call is attempted. As defined in H.225.0, an endpoint shall register with a single Gatekeeper via a Registration Request (RRQ) message. The Gatekeeper then responds with either a Registration Confirmation (RCF) or a Registration Reject (RRJ) to indicate the registration status. Each mobile terminal has a finite registration life and requires a reregistration after the expiration. We will defer the discussion of registration during roaming to Sec. 3.3. Note that authentication and other security issues are important to registration as well. These issues are outside the scope of this paper.

\subsection{Call establishment}

The call made between a POTS phone and an H.323 terminal can be in either direction, i.e., from a POTS phone to an H.323 terminal or an H.323 terminal to a POTS phone.

(1) ITG (caller) to H.323 terminal (callee)

Registration should be performed first before any call is attempted. Assume each H.323 terminal is registered and managed under a single Gatekeeper in the 
respective zone. When a call is destined to an $\mathrm{H} .323$ terminal (either stationary or mobile) from the ITG, a paging message of location discovery for the H.323 terminal is sent by the ITG. The caller has no idea as to whether the callee is stationary or mobile. If the called H.323 terminal is a fixed IP host, it always stays in the home zone, under the management of the home Gatekeeper. If the called H.323 terminal is a mobile IP host and has roamed to a foreign zone, it is under the management of a foreign Gatekeeper. The caller, however, does not know the identity and location of the corresponding Gatekeeper of the callee. As defined in H.225.0, when an endpoint would like to determine the contact information of the other endpoint, it may multicast a Location Request (LRQ) message to the Gatekeeper well-known discovery multicast address. If the endpoint is registered, then the corresponding Gatekeeper responds with the Location Confirmation (LCF) message containing the contact information of the endpoint; otherwise a Location Reject (LRJ) is returned.

Therefore, this paging message is sent to the Gatekeeper well-known discovery multicast address with an LRQ message so that all Gatekeepers are able to receive the page. This is similar to broadcasting a paging message to all base stations in the cellular phone systems. Only the corresponding Gatekeeper acknowledges the paging message with the callee's IP address in an LCF message, either the real IP address (from the home Gatekeeper) or the care-of address (from a foreign Gatekeeper). Once the callee is located, the connection is established. The caller initiates the connection establishment through the normal setup procedure for a point to point conference. The call signaling of connection establishment is shown in Fig. 4 (a). Note that CID means Conference ID, and TA, Transport Address.

\section{(2) H.323 terminal (caller) to ITG (callee)}

When an H.323 terminal calls a POTS phone, no paging message for location discovery is required, but the address translation for ITG location lookup performed through the directory service by the corresponding Gatekeeper is necessary. ITG location discovery is another important issue for the success of Internet telephony. It is outside the scope of this paper. A variety of strategies discussed in [9], such as SLP [10] and LDAP [11], may be used for locating the ITG. Once the translated address of corresponding ITG is return, the normal creation procedure for a point to point conference is performed. The call signaling of connection establishment is shown in Fig. 4 (b). Note that the address translation for the ITG is not shown in Fig. 4 (b).

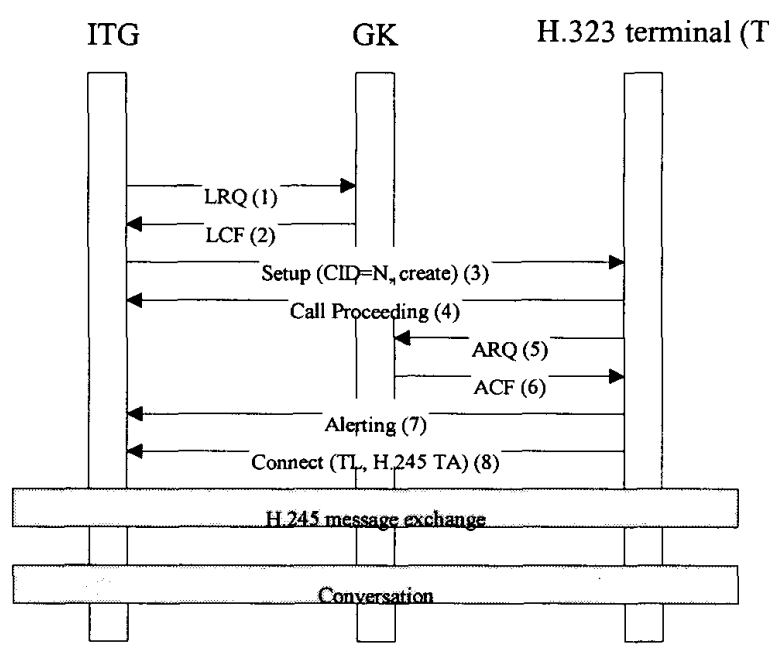

(a) ITG to H.323 terminal

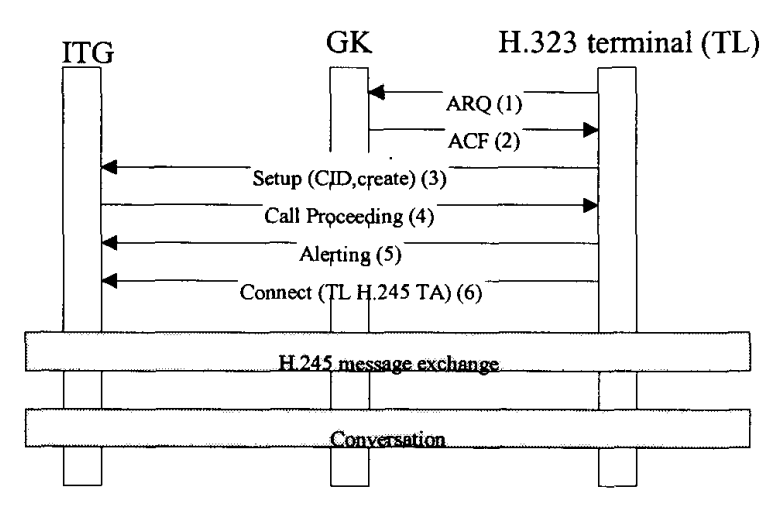

(b) H.323 terminal to ITG

Fig. 4: Call signaling for call establishment

\subsection{Roaming and handoff}

When an H.323 terminal is roaming, it may be in intra-zone roaming or inter-zone roaming. Handoff occurs when the terminal TL1 at the subnet 1 roams to the subnet2 as shown in Fig. 2. As mentioned earlier, a handoff can be handled as dynamically joining and leaving an ad hoc multipoint conference. With ad hoc multipoint conference expansions, there are two 
alternatives for a new endpoint to join the conference through the $\mathrm{MC}$ (Multipoint Controller): request-to-join (by requesting on its own) and invite-to-join (being invited by any participant). Once the new endpoint TL2 has joined the conference in either case, TL1 leaves the conference without affecting the ongoing call. In the following, only the request-to-join approach is demonstrated. Invite-to-join can be performed in a similar manner.

For intra-zone roaming, a handoff can be handled using normal procedures for a new endpoint joining an ad hoc multipoint conference, followed by an old endpoint leaving the conference. For inter-zone roaming, Gatekeeper discovery should be performed first. This is necessary because the visiting mobile host has no idea as to where the foreign Gatekeeper in the foreign zone is. As mentioned in Sec. 3.1, there are two different ways for Gatekeeper discovery: (1) Gatekeeper advertisement, or (2) Gatekeeper solicitation. Once the foreign Gatekeeper has been identified through the discovery process, registration occurs. During registration, location update takes place in which the foreign Gatekeeper informs the home Gatekeeper the care-of address of the mobile host and stores such location information in its local directory, and the home Gatekeeper instructs the previous foreign Gatekeeper to remove the entry of the mobile terminal. Then handoff is handled the same way as in intra-zone roaming, i.e., with the normal procedures for a new endpoint joining an ad hoc multipoint conference, followed by the departure of an old endpoint. In H.225.0, an endpoint issues a Disengage Reject (DRQ) message to terminate a call. The Gatekeeper then returns a Disengage Confirmation (DCF) message. To cancel a registration, an endpoint may either (1) send an Unregister Request (URQ) message to the Gatekeeper, which then responds with either an Unregister Confirmation (UCF) message or an Unregister Reject (URJ) message, or (2) wait until the registration lifetime expires. Of course, H.245 logical channels for the connection should be closed first. Therefore, to break the old connection, the mobile host should de-register from the previous Gatekeeper with a DRQ and URQ messages to complete the handoff.

Signaling procedures for roaming and handoff handled by the "request-to-join" conference approach are depicted in Fig. 5, equivalent to ad hoc multipoint conference join and departure. Note that in Fig. 5, (1) to
(4) are for registration to the new Gatekeeper GK2 and (15)-(16) are for de-registration from the previous Gatekeeper GK1. Only the Gatekeeper solicitation for discovery is shown, without depicting location update among Gatekeepers. The rest of the procedures remain the same as in Fig. 5(a).

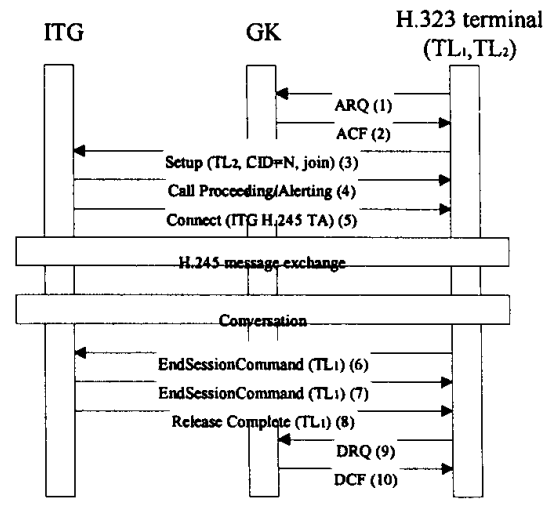

(a) Intra-zone roaming

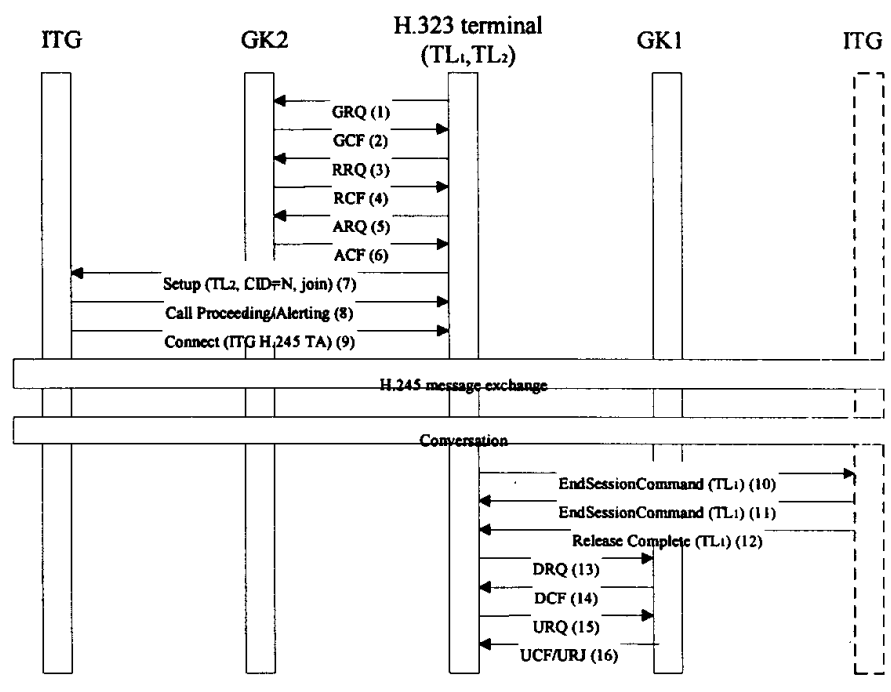

(b) Inter-zone roaming

Fig. 5: Call signaling for handoff

\section{Concluding remarks}

We have proposed an approach to realize mobile Internet telephony service from the mobile extensions to ITU-T Rec. H.323. The system architecture has been described, and the corresponding mobility management has been presented. No extra system entities are required to support host mobility. We try to reuse the existing protocols in IP networks and stick to the control messages 
and functions defined in Internet telephony mechanism as much as we can, and show how to seamlessly integrate mobility features into H.323 compliant IP telephony.

The proposed approach combines the characteristics of circuit-switched cellular phone systems and packetswitched Mobile IP mechanism with Internet telephony, thereby allowing connection-oriented mobile telephone services over IP-based networks. Through the proposed mobile Internet telephony system, real-time voice services for both stationary and mobile IP hosts are supported. The optimization in the routing protocol in Mobile IP [12] is implicitly offered in the proposed approach, because the paging message for host location lookup is broadcast to all Gatekeepers, followed by the location information returned by the corresponding Gatekeeper for further call routing. As a result, tt enables the service redirection to be completely handled in the application layer, thereby realizing mobile IP telephony services with IP rather than with the Mobile IP mechanism.

The most striking result is that we have transformed host mobility management into the ad hoc multipoint conference manipulation. The key operations for mobility management are summarized as follows. (1) Registration can be achieved through the conference registration process, plus one extra message defined for Gatekeeper advertisement. (2) Connection establishment and termination for mobile Internet telephony service can utilize the procedures for creation and termination of a point to point conference, plus location discovery for the mobile terminal. (3) Handoff handling for global roaming can be solved through the procedures of dynamic join and departure of the conference during a call, plus registration and location update. Through the proposed approach, the host mobility can be seamlessly included as a value-added feature in the widely growing H.323 compliant Internet telephony systems.

\section{References}

[1] ITU-T Rec. H.323v2, "Packet Based Multimedia Communications Systems," Mar. 1997

[2] G. A. Thom, "H.323: the Multimedia Comm. Standard for Local Area Networks," IEEE Comm.
Magazine, pp. 52-56, Dec. 1996

[3] H. Schulzrinne et al. "RTP: A Transport Protocol for Real-Time applications," RFC 1889, Nov. 1995.

[4] S. Redl and M. Weber, "An Introduction to GSM," Artech House, 1995

[5] C. Perkins, "IP Mobility Support," RFC 2002, Oct. 1996, available at $\mathrm{ftp}: / / \mathrm{ds}$.intenic.net/rfc/rfc2002.txt

[6] R. Droms, "Dynamic Host Configuration Protocol," RFC 2131, Mar. 1997

[7] ITU-T Rec. H.225.0, "Media Stream Packetization and Synchronization for Visual Telephone Systems on Non-Guaranteed Quality of Service LANs"

[8] ITU-T Rec. H.245, "Control Protocol for Multimedia Communication," Mar. 1996

[9] J. Rosenberg and H. Schulzrinne, "Internet Telephony Gateway Location,” Proc. Infocom'98

[10] J. Veizades et al. "Service location Protocol," RFC 2165, June 1997

[11] W. Yeong, T. Howes, and S. Kille, "Lightweight Directory Access Protocol," RFC 1777, Mar. 1995

[12] A. Myles et al. "A Mobile Host Protocol Supporting Route Optimization and Authentication," IEEE Journal on Selected Areas in Communications, Vol. 13, Vol. 5, pp. 839-849, June 1995 